\title{
Examination of Haematotoxicity of Fixed-Dose Highly Active Antiretroviral Drug in Albino Wistar Rats
}

\author{
Thomas Nubila, ${ }^{1}$ Ernest O. Ukaejiofo, ${ }^{1}$ Nkoyo I. Nubila, ${ }^{2}$ and Godfrey I. Okorie ${ }^{3}$ \\ ${ }^{1}$ Department of Medical Laboratory Sciences, Faculty of Health Sciences and Technology, College of Medicine, University of Nigeria, \\ Enugu Campus, Enugu State, Enugu 400001, Nigeria \\ ${ }^{2}$ Department of Pharmacology and Therapeutics, Faculty of Medical Sciences, College of Medicine, University of Nigeria, \\ Enugu Campus, PMB 01129, Enugu State, Enugu 400001, Nigeria \\ ${ }^{3}$ Department of Haematology, University of Nigeria Teaching Hospital (UNTH), Ituku-Ozalla, PMB 01129, Enugu State, \\ Enugu 400001, Nigeria
}

Correspondence should be addressed to Thomas Nubila, tnubila@gmail.com

Received 16 September 2012; Accepted 18 October 2012

Academic Editors: G. Biala and R. Fantozzi

Copyright (C) 2012 Thomas Nubila et al. This is an open access article distributed under the Creative Commons Attribution License, which permits unrestricted use, distribution, and reproduction in any medium, provided the original work is properly cited.

Highly active antiretroviral therapy (HAART) is considered toxic and has other life-threatening side effects. Our aim was to evaluate the haematotoxic effects of lamivudine, zidovudine, and nevirapine fixed-dose combinations in Albino Wistar rats. Fifty (50) three (3) months old male Albino Wistar rats weighing between 200 and $250 \mathrm{~g}$ were randomly assigned to five (5) groups (A, B, C, D, and E). Group A served as control. Two (2 mLs) of venous blood was aseptically collected on Days 5, 10, 15, 20, and 25 of treatment. Red blood cell (RBC) mean value recorded statistically significant increase $(P<0.05)$ in groups $B$ and $C$ when compared with the control group on Day 5 . However, there was a statistically significant decrease $(P<0.05)$ in RBC, haemoglobin concentration $(\mathrm{Hb})$, packed cell volume (PCV), and some red cell indices on Day 10. In addition there was no statistically significant difference $(P>0.05)$ in all the parameters evaluated when the test group was compared with the control on Day 25. Furthermore, there was a time-related statistically significant increase $(P<0.05)$ in the two major blood cells-RBC and platelet counts. From the result of this present study, it can be concluded that HAART when administered in fixed-dose combinations have no subacute haematotoxic effects.

\section{Introduction}

Antiretroviral drugs are medication for treatment of infection by retroviruses, primarily human immunodeficiency virus (HIV). When several such drugs, typically three or four, are taken in combination, the approach is known as highly active antiretroviral therapy, or HAART. The American National Institute of Health and other organizations recommend offering antiretroviral treatment to all patients with AIDS. Because of the complexity of selecting and following a regimen, the severity of the side effects and the importance of compliance to prevent viral resistance, such organizations emphasize the importance of involving patients in therapy choices and recommend analyzing the risks and the potential benefits to patients with low viral loads [1].
There is no doubt that HAART has been the most important progress in the therapy of HIV-infected patients in the last decade. A growing number of observations suggest that the beneficial effects of HAART also include improvement of HIV-related haematological complications [2]. Current guidelines for treatment of human immune-deficiency virus (HIV) infection recommend the combination of three antiretroviral agents, two reverse transcriptase inhibitors (RTIs) plus one protease inhibitor, or the association of three RTIs $[1,3]$. These regimens of HAART have dramatically reduced the morbidity and mortality of HIV infection [2].

The values of haematologic parameters are affected by a number of factors even in apparently healthy populations. These factors include age, sex, ethnic background, body build, and social, nutritional, and environmental factors, 
especially altitude [4-6]. It has also been shown in several studies that some of the heamatological parameters exhibit considerable variations at different periods of life and disease conditions [7-9]. Although HAART has dramatically improved survival in HIV-infected patients, precautions still need to be taken to prevent HAART-related haematotoxicity and other life-threatening side-effects. Due to the fact that there are no available preclinical safety data on the treatment with the combinations of lamivudine, zidovudine, and nevirapine in animals, the aim of the present study was to examine the potential haematotoxicity of combinations of lamivudine, zidovudine, and nevirapine in Albino Wistar rats.

\section{Materials and Methods}

2.1. Drugs and Sources. The three (3) fixed-dose antiretroviral combination drugs used for the study were obtained from one of the accredited United States Presidential Emergency Plan for AIDS Relief (PEPFAR) centers in Enugu, Enugu State, Nigeria. The HAART considered in the study was a fixed-dose combinations (FDCs) of lamividine (150 mg), zidovudine $(300 \mathrm{mg})$, and nevarapine $(200 \mathrm{mg})$ per tablet. The drug was manufactured by Aurobindo Pharma Limited, Unit III, survey No. 313, Bachupally village, Quthubullapur Mandal, Ranga Reddy District (A.P) India.

2.2. Animal Care and Handling. This was according to the guidelines issued by World Health Organization (WHO), Geneva, Switzerland and the Indian National Science Academy (INSA), New Delhi, India on the care and use of laboratory animals. The Albino Wistar rats which were three (3) months old and weighing 200-250 g were selected from the colony maintained under the controlled conditions of temperature $\left(33 \pm 2^{\circ} \mathrm{C}\right)$, humidity, and light (12 hours of light and dark) in the Animal House of University of Nigeria, Enugu Campus (UNEC), Enugu, Enugu State, Nigeria. The animals had free access to food (Standard pellet diet, starter feed) and clean tap water. The animals were housed in standard environmental conditions in wire-mesh-bottomed stainless steel cages.

\section{Experimental Design}

Fifty (50) three (3) months old male Albino Wistar rats weighing between 200-250 g were randomly assigned to five (5) groups (A, B, C, D, and E) according to similar body weight with ten (10) animals per group. Groups B, C, D, and E received graded doses (3, 6, 12, and $24 \mathrm{mg} / \mathrm{Kg}$ body weight, resp.) once daily for twenty five (25) days. Group A served as control. All administrations were by oral intubation.

\subsection{Blood Sample Collection and Analysis. Two (2 mLs) of} venous blood was aseptically collected on Days 5, 10, 15, 20 , and 25 of treatment after overnight fasting from each animal into tripotassium ethylene diamine tetra acetic acid ( $\mathrm{K}_{3}$ EDTA) anticoagulant bottle. This was immediately mixed by gentle inversion and used in the determination of the haematological profile using a haematology auto analyser (Sysmex KX-2IN) following the manufacturer's guidelines. The samples were analysed within two hours of collection. Two (2) animals were bled painlessly under chloroform anesthesia by ocular puncture through the retro-bulber plexus of the medial canthus.

3.2. Data Analysis. Data were analysed using Students' " $t$ "-test and one-way analysis of Variance (ANOVA) to determine significant differences between means. $P<0.05$ was regarded as significant.

\section{Results}

Red blood cells (RBCs) count mean value recorded statistically significant increase $(P<0.05)$ in groups B $(3 \mathrm{mg} / \mathrm{kg}$ body weight) and group $\mathrm{C}(6 \mathrm{mg} / \mathrm{kg}$ body weight $)$ when compared with the control group. However, total white blood cell (WBC) count showed statistically significant decrease $(P<0.01)$ in group $\mathrm{B}$ when compared with the control group. RBC and mean cell haemoglobin $(\mathrm{MCH})$ mean values revealed dose dependent statistically significant decrease $(P<0.001$ and $P<0.05)$, respectively, in the one-way analysis of variance (ANOVA) when groups B, C, D, and E were compared on Day 5 (Table 1).

However, RBC, haemoglobin ( $\mathrm{Hb})$, and packed cell volume $(\mathrm{PCV})$ recorded statistically significant decrease $(P<$ $0.05)$ in group $\mathrm{E}(24 \mathrm{mg} / \mathrm{kg}$ body weight $)$ when compared with the control group. Also, mean cell volume $(\mathrm{MCV})$ and MCHC revealed statistically significant decrease $(P<0.001$ and $P<0.05)$ when compared with the control group. Neutrophil count showed a statistically significant increase $(P<$ 0.05 ) in group B when compared with the control group, while lymphocyte count recorded statistically significant decrease $(P<0.05)$ when compared with the control group. MCV revealed a dose-dependent statistically significant increase $(P<0.05)$ in the one way ANOVA when groups $B$, C, D, and E were compared on Day 10 (Table 2).

Only platelet count mean value recorded a dose-dependent statistically significant increase $(P<0.05)$ when all the test groups were compared on Day 15 (Table 3 ).

In addition, there was no statistically significant difference $(P>0.05)$ in all the parameters evaluated when the test groups was compared with the control group on Day 20 (Table 4).

However, only total WBC showed a statistically significant decrease $(P<0.05)$ in groups $\mathrm{D}(12 \mathrm{mg} / \mathrm{kg}$ body weight $)$ and $\mathrm{E}$ when compared with the control group on Day 25 (Table 5).

Furthermore, in group $\mathrm{C}, \mathrm{RBC}$, and $\mathrm{Hb}$ mean values demonstrated a time-dependent statistically significant increase $(P<0.05)$. Similarly, platelet and lymphocyte count showed a statistically significant increase $(P<0.01$ and $P<$ 0.001), respectively. However, MCHC and neutrophil mean values showed a time-dependent statistically significant decrease $(P<0.01$ and $P<0.001$, resp. $)$ in all the test groups (Table 6). 
TABLE 1: Comparison of the mean \pm S.E of the haematological parameters after 5 days of HAART administration with the control group.

\begin{tabular}{|c|c|c|c|c|c|}
\hline Parameter & $\begin{array}{l}\text { Group A } \\
0 \mathrm{mg} / \mathrm{Kg}\end{array}$ & $\begin{array}{l}\text { Group B } \\
3 \mathrm{mg} / \mathrm{Kg}\end{array}$ & $\begin{array}{l}\text { Group C } \\
6 \mathrm{mg} / \mathrm{Kg}\end{array}$ & $\begin{array}{l}\text { Group D } \\
12 \mathrm{mg} / \mathrm{Kg}\end{array}$ & $\begin{array}{c}\text { Group E } \\
24 \mathrm{mg} / \mathrm{Kg}\end{array}$ \\
\hline $\mathrm{RBC}\left(\times 10^{12} / \mathrm{L}\right)$ & $6.05 \pm 0.30$ & $6.30 \pm 0.25^{*}$ & $7.23 \pm 0.13^{*}$ & $5.98 \pm 0.005$ & $6.38 \pm 0.15^{\beta \beta \beta}$ \\
\hline $\mathrm{HB}(\mathrm{g} / \mathrm{dL})$ & $11.95 \pm 0.45$ & $11.65 \pm 0.65$ & $12.55 \pm 0.25$ & $11.60 \pm 0.20$ & $11.80 \pm 0.50$ \\
\hline $\mathrm{PCV}(\mathrm{L} / \mathrm{L})$ & $38.50 \pm 1.50$ & $37.00 \pm 2.00$ & $39.00 \pm 1.00$ & $36.0 \pm 2.00$ & $36.50 \pm 1.50$ \\
\hline MCHC (g/dL) & $31.25 \pm 0.05$ & $31.60 \pm 0.40$ & $32.00 \pm 1.20$ & $32.5 \pm 0.50$ & $32.00 \pm 2.00$ \\
\hline MCV (fL) & $63.50 \pm 2.50$ & $58.60 \pm 3.60$ & $57.50 \pm 0.50$ & $60.00 \pm 2.00$ & $58.0 \pm 1.00$ \\
\hline $\mathrm{MCH}(\mathrm{pg})$ & $19.95 \pm 1.05$ & $18.70 \pm 0.70$ & $17.30 \pm 0.10$ & $19.35 \pm 0.05$ & $18.55 \pm 0.45^{\beta}$ \\
\hline RETICS (\%) & $1.00 \pm 0.10$ & $1.05 \pm 0.15$ & $1.30 \pm 0.30$ & $1.25 \pm 0.15$ & $0.80 \pm 0.10$ \\
\hline PLAT $\left(\times 10^{9} / \mathrm{L}\right)$ & $446.50 \pm 131.50$ & $559.50 \pm 98.50$ & $571.00 \pm 72.00$ & $571.50 \pm 74.50$ & $582.50 \pm 6.50$ \\
\hline $\mathrm{WBC}\left(\times 10^{9} / \mathrm{L}\right)$ & $10.95 \pm 0.15$ & $5.90 \pm 0.10^{* *}$ & $6.85 \pm 1.95$ & $7.75 \pm 74.50$ & $9.10 \pm 4.20$ \\
\hline NEUT (\%) & $33.00 \pm 7.00$ & $31.50 \pm 12.50$ & $20.00 \pm 4.00$ & $60.00 \pm 2.00$ & $30.5 \pm 8.50$ \\
\hline LYMPH (\%) & $65.00 \pm 7.00$ & $67.00 \pm 11.00$ & $78.50 \pm 3.50$ & $68.00 \pm 8.00$ & $67.50 \pm 6.50$ \\
\hline EOS (\%) & $0.00 \pm 0.00$ & $0.5 \pm 0.50$ & $0.50 \pm 0.50$ & $0.00 \pm 0.00$ & $1.00 \pm 1.00$ \\
\hline MONO (\%) & $2.00 \pm 1.00$ & $1.00 \pm 1.00$ & $1.00 \pm 1.00$ & $0.00 \pm 0.00$ & $1.00 \pm 1.00$ \\
\hline BAS (\%) & $0.00 \pm 0.00$ & $0.00 \pm 0.00$ & $0.00 \pm 0.00$ & $0.00 \pm 0.00$ & $0.00 \pm 0.00$ \\
\hline
\end{tabular}

RBC: red blood cell, HB: haemoglobin concentration, PCV: packed cell volume, MCHC: mean cell haemoglobin concentration, MCV: mean cell volume, MCH: mean cell haemoglobin, RETICS: reticulocytes, PLAT: platelets, WBC: white blood cell, NEUT: neutrophils, LYMPH: lymphocytes, EOS: eosinophils, BAS: basophils.

*Compared with the control group $* P<0.05,{ }^{* *} P<0.01$.

$\beta$ One way ANOVA for groups B, C, D, and $\mathrm{E}, \beta \beta \beta P<0.001$.

TABLE 2: Comparison of the mean \pm S.E of the haematological parameters after 10 days of HAART administration with the control group.

\begin{tabular}{|c|c|c|c|c|c|}
\hline Parameter & $\begin{array}{l}\text { Group A } \\
0 \mathrm{mg} / \mathrm{Kg}\end{array}$ & $\begin{array}{l}\text { Group B } \\
3 \mathrm{mg} / \mathrm{Kg}\end{array}$ & $\begin{array}{l}\text { Group C } \\
6 \mathrm{mg} / \mathrm{Kg}\end{array}$ & $\begin{array}{l}\text { Group D } \\
12 \mathrm{mg} / \mathrm{Kg}\end{array}$ & $\begin{array}{c}\text { Group E } \\
24 \mathrm{mg} / \mathrm{Kg}\end{array}$ \\
\hline $\mathrm{RBC}\left(\times 10^{12} / \mathrm{L}\right)$ & $6.34 \pm 0.22$ & $7.41 \pm 1.05$ & $6.89 \pm 0.14$ & $7.49 \pm 1.08$ & $4.92 \pm 0.20^{*}$ \\
\hline $\mathrm{HB}(\mathrm{g} / \mathrm{dL})$ & $11.60 \pm 0.40$ & $12.95 \pm 1.75$ & $12.55 \pm 0.45$ & $13.15 \pm 1.95$ & $9.40 \pm 0.10^{*}$ \\
\hline $\mathrm{PCV}(\mathrm{L} / \mathrm{L})$ & $40.50 \pm 1.50$ & $44.50 \pm 6.50$ & $42.50 \pm 1.50$ & $44.00 \pm 8.00$ & $33.50 \pm 0.50^{*}$ \\
\hline $\operatorname{MCHC}(\mathrm{g} / \mathrm{dL})$ & $23.70 \pm 0.30$ & $29.10 \pm 0.10$ & $29.70 \pm 0.30$ & $30.00 \pm 1.00$ & $28.00 \pm 1.00$ \\
\hline $\mathrm{MCV}(\mathrm{fL})$ & $64.20 \pm 0.20$ & $60.20 \pm 0.10^{* *}$ & $62.00 \pm 1.00$ & $59.00 \pm 2.00$ & $68.50 \pm 1.50^{\beta}$ \\
\hline $\mathrm{MCH}(\mathrm{pg})$ & $18.30 \pm 0.20$ & $17.20 \pm 0.10^{*}$ & $18.15 \pm 0.25$ & $17.75 \pm 0.15$ & $19.10 \pm 1.00$ \\
\hline RETICS (\%) & $1.35 \pm 0.05$ & $1.35 \pm 0.35$ & $1.15 \pm 0.15$ & $1.50 \pm 0.10$ & $1.35 \pm 0.15$ \\
\hline PLAT $\left(\times 10^{9} / \mathrm{L}\right)$ & $431.50 \pm 68.50$ & $328.00 \pm 55.00$ & $370.00 \pm 232.00$ & $219.00 \pm 73.00$ & $284.00 \pm 223$ \\
\hline $\mathrm{WBC}\left(\times 10^{9} / \mathrm{L}\right)$ & $14.05 \pm 3.55$ & $6.70 \pm 0.50$ & $9.30 \pm 1.10$ & $6.60 \pm 1.20$ & $8.80 \pm 3.00$ \\
\hline NEUT (\%) & $15.00 \pm 3.00$ & $33.50 \pm 2.50^{*}$ & $24.50 \pm 3.50$ & $24.00 \pm 12.00$ & $27.00 \pm 1.00$ \\
\hline LYMPH (\%) & $85.00 \pm 3.00$ & $65.50 \pm 1.50^{*}$ & $75.50 \pm 3.50$ & $76.00 \pm 12.00$ & $73.00 \pm 1.00$ \\
\hline EOS $(\%)$ & $0.00 \pm 0.00$ & $0.00 \pm 0.00$ & $0.50 \pm 0.50$ & $0.00 \pm 0.00$ & $0.00 \pm 0.00$ \\
\hline MONO (\%) & $0.00 \pm 0.00$ & $1.00 \pm 1.00$ & $0.00 \pm 0.00$ & $0.00 \pm 0.00$ & $0.00 \pm 0.00$ \\
\hline BAS (\%) & $0.00 \pm 0.00$ & $0.00 \pm 0.00$ & $0.00 \pm 0.00$ & $0.00 \pm 0.00$ & $0.00 \pm 0.00$ \\
\hline
\end{tabular}

RBC: red blood cell, HB: haemoglobin concentration, PCV: packed cell volume, MCHC: mean cell haemoglobin concentration, MCV: mean cell volume, MCH: mean cell haemoglobin, RETICS: reticulocytes, PLAT: platelets, WBC: white blood cell, NEUT: neutrophils, LYMPH: lymphocytes, EOS: eosinophils, BAS: basophils.

*Compared with the control group $* P<0.05,{ }^{* *} P<0.01$.

$\beta$ One way ANOVA for groups $\mathrm{B}, \mathrm{C}, \mathrm{D}$, and $\mathrm{E}^{\beta} P<0.05$.

\section{Discussion}

Although HAART has dramatically improved survival in HIV patients, precautions still need to be taken to prevent HAART-related haematotoxicity and other life threatening side effects. In this study, red blood cell (RBC) mean value recorded statistical significant increase $(P<0.05)$ in groups $\mathrm{B}$ and $\mathrm{C}$ when compared with the control group on Day 5. This could most likely be due to the stimulatory effect of HAART on haemopoiesis since the total white blood cell (WBC) count showed a statistical significant decrease in the same group B. However, one may be tempted to suggest that 
TABLE 3: Comparison of the mean \pm S.E of the haematological parameters after 15 days of HAART administration with the control group.

\begin{tabular}{|c|c|c|c|c|c|}
\hline Parameter & $\begin{array}{l}\text { Group A } \\
0 \mathrm{mg} / \mathrm{Kg}\end{array}$ & $\begin{array}{l}\text { Group B } \\
3 \mathrm{mg} / \mathrm{Kg}\end{array}$ & $\begin{array}{l}\text { Group C } \\
6 \mathrm{mg} / \mathrm{Kg}\end{array}$ & $\begin{array}{l}\text { Group D } \\
12 \mathrm{mg} / \mathrm{Kg}\end{array}$ & $\begin{array}{c}\text { Group E } \\
24 \mathrm{mg} / \mathrm{Kg}\end{array}$ \\
\hline $\mathrm{RBC}\left(\times 10^{12} / \mathrm{L}\right)$ & $8.11 \pm 0.51$ & $6.74 \pm 1.03$ & $9.14 \pm 0.61$ & $7.77 \pm 0.40$ & $6.63 \pm 0.26$ \\
\hline $\mathrm{HB}(\mathrm{g} / \mathrm{dL})$ & $14.25 \pm 1.25$ & $12.60 \pm 0.50$ & $14.55 \pm 1.15$ & $13.95 \pm 0.35$ & $12.35 \pm 0.35$ \\
\hline $\mathrm{PCV}(\mathrm{L} / \mathrm{L})$ & $55.00 \pm 4.00$ & $44.50 \pm 6.50$ & $57.40 \pm 5.40$ & $51.50 \pm 0.50$ & $46.50 \pm 0.50$ \\
\hline $\mathrm{MCHC}(\mathrm{g} / \mathrm{dL})$ & $25.70 \pm 0.50$ & $25.95 \pm 0.35$ & $25.50 \pm 0.50$ & $26.95 \pm 0.95$ & $26.50 \pm 1.10$ \\
\hline $\operatorname{MCV}(\mathrm{fL})$ & $68.50 \pm 0.50$ & $66.65 \pm 0.65$ & $62.95 \pm 1.45$ & $66.80 \pm 2.80$ & $70.35 \pm 1.85$ \\
\hline $\mathrm{MCH}(\mathrm{pg})$ & $17.55 \pm 0.45$ & $17.30 \pm 0.40$ & $15.90 \pm 0.20$ & $18.05 \pm 1.35$ & $17.40 \pm 0.10$ \\
\hline RETICS (\%) & $1.45 \pm 0.25$ & $1.05 \pm 0.15$ & $1.60 \pm 0.20$ & $1.30 \pm 0.30$ & $1.10 \pm 0.20$ \\
\hline PLAT $\left(\times 10^{9} / \mathrm{L}\right)$ & $569.50 \pm 229.50$ & $383.00 \pm 16.00$ & $800.50 \pm 20.50$ & $660.50 \pm 134.50$ & $844.00 \pm 45.00^{\beta}$ \\
\hline $\mathrm{WBC}\left(\times 10^{9} / \mathrm{L}\right)$ & $8.85 \pm 1.05$ & $5.85 \pm 1.85$ & $5.95 \pm 1.05$ & $9.35 \pm 1.55$ & $9.80 \pm 0.40$ \\
\hline NEUT (\%) & $21.50 \pm 1.50$ & $14.00 \pm 6.00$ & $27.00 \pm 3.00$ & $23.50 \pm 2.50$ & $22.50 \pm 0.50$ \\
\hline LYMPH (\%) & $78.50 \pm 1.50$ & $86.00 \pm 6.00$ & $72.00 \pm 4.00$ & $76.50 \pm 2.50$ & $77.50 \pm 0.50$ \\
\hline EOS $(\%)$ & $0.00 \pm 0.00$ & $0.00 \pm 0.00$ & $0.00 \pm 0.00$ & $0.00 \pm 0.00$ & $0.00 \pm 0.00$ \\
\hline MONO (\%) & $0.00 \pm 0.00$ & $0.00 \pm 0.00$ & $1.00 \pm 1.00$ & $0.00 \pm 0.00$ & $0.00 \pm 0.00$ \\
\hline BAS (\%) & $0.00 \pm 0.00$ & $0.00 \pm 0.00$ & $0.00 \pm 0.00$ & $0.00 \pm 0.00$ & $0.00 \pm 0.00$ \\
\hline
\end{tabular}

RBC: red blood cell, HB: haemoglobin concentration, PCV: packed cell volume, MCHC: mean cell haemoglobin concentration, MCV: mean cell volume, MCH: mean cell haemoglobin, RETICS: reticulocytes, PLAT: platelets, WBC: white blood cell, NEUT: neutrophils, LYMPH: lymphocytes, EOS: eosinophils, BAS: basophils.

$\beta$ One way ANOVA for groups $\mathrm{B}, \mathrm{C}, \mathrm{D}$, and $\mathrm{E} \beta \mathrm{P}<0.05$.

TABLE 4: Comparison of the mean \pm S.E of the haematological parameters after 20 days of HAART administration with the control group.

\begin{tabular}{|c|c|c|c|c|c|}
\hline Parameter & $\begin{array}{l}\text { Group A } \\
0 \mathrm{mg} / \mathrm{Kg}\end{array}$ & $\begin{array}{l}\text { Group B } \\
3 \mathrm{mg} / \mathrm{Kg}\end{array}$ & $\begin{array}{l}\text { Group C } \\
6 \mathrm{mg} / \mathrm{Kg}\end{array}$ & $\begin{array}{l}\text { Group D } \\
12 \mathrm{mg} / \mathrm{Kg}\end{array}$ & $\begin{array}{c}\text { Group E } \\
24 \mathrm{mg} / \mathrm{Kg}\end{array}$ \\
\hline $\mathrm{RBC}\left(\times 10^{12} / \mathrm{L}\right)$ & $7.80 \pm 1.20$ & $8.42 \pm 0.08$ & $7.86 \pm 0.31$ & $6.76 \pm 0.93$ & $7.40 \pm 0.02$ \\
\hline $\mathrm{HB}(\mathrm{g} / \mathrm{dL})$ & $13.80 \pm 1.60$ & $15.60 \pm 0.50$ & $13.70 \pm 0.40$ & $12.71 \pm 1.30$ & $13.15 \pm 0.05$ \\
\hline $\mathrm{PCV}(\mathrm{L} / \mathrm{L})$ & $53.50 \pm 6.50$ & $56.95 \pm 0.95$ & $51.00 \pm 1.00$ & $47.75 \pm 5.75$ & $50.60 \pm 1.40$ \\
\hline $\mathrm{MCHC}(\mathrm{g} / \mathrm{dL})$ & $26.05 \pm 0.05$ & $27.55 \pm 1.45$ & $26.70 \pm 0.40$ & $26.80 \pm 0.60$ & $26.15 \pm 0.85$ \\
\hline MCV (fL) & $70.55 \pm 1.65$ & $67.60 \pm 0.50$ & $65.30 \pm 1.70$ & $70.70 \pm 0.70$ & $68.70 \pm 1.70$ \\
\hline $\mathrm{MCH}(\mathrm{pg})$ & $18.25 \pm 0.55$ & $18.55 \pm 0.75$ & $17.45 \pm 0.15$ & $18.90 \pm 0.70$ & $17.80 \pm 0.10$ \\
\hline RETICS (\%) & $1.35 \pm 0.15$ & $1.20 \pm 0.20$ & $0.95 \pm 0.05$ & $1.50 \pm 0.05$ & $1.15 \pm 0.25$ \\
\hline $\operatorname{PLAT}\left(\times 10^{9} / \mathrm{L}\right)$ & $534.00 \pm 95.50$ & $516.00 \pm 37.00$ & $787.50 \pm 1.50$ & $941.00 \pm 320.00$ & $958.00 \pm 166.00$ \\
\hline $\mathrm{WBC}\left(\times 10^{9} / \mathrm{L}\right)$ & $7.20 \pm 0.60$ & $7.95 \pm 1.55$ & $7.65 \pm 2.05$ & $6.95 \pm 1.65$ & $11.90 \pm 2.40$ \\
\hline NEUT (\%) & $28.00 \pm 14.00$ & $24.00 \pm 4.00$ & $25.50 \pm 2.50$ & $17.50 \pm 4.50$ & $7.50 \pm 0.50$ \\
\hline LYMPH (\%) & $72.00 \pm 14.00$ & $76.00 \pm 4.00$ & $74.5 \pm 2.50$ & $81.00 \pm 5.00$ & $92.50 \pm 0.50$ \\
\hline EOS (\%) & $0.00 \pm 0.00$ & $0.00 \pm 0.00$ & $0.00 \pm 0.00$ & $0.00 \pm 0.00$ & $0.00 \pm 0.00$ \\
\hline MONO (\%) & $0.00 \pm 0.00$ & $0.00 \pm 0.00$ & $0.00 \pm 0.00$ & $1.50 \pm 0.50$ & $0.00 \pm 0.00$ \\
\hline BAS (\%) & $0.00 \pm 0.00$ & $0.00 \pm 0.00$ & $0.00 \pm 0.00$ & $0.00 \pm 0.00$ & $0.00 \pm 0.00$ \\
\hline
\end{tabular}

RBC: red blood cell, HB: haemoglobin concentration, PCV: packed cell volume, MCHC: mean cell haemoglobin concentration, MCV: mean cell volume, MCH: mean cell haemoglobin, RETICS: reticulocytes, PLAT: platelets, WBC: white blood cell, NEUT: neutrophils, LYMPH: lymphocytes, EOS: eosinophils, BAS: basophils.

the drug had an inhibitory effect on haemopoiesis in general and erythropoiesis in particular as shown by a statistically significant decrease $(P<0.05)$ in RBC, haemoglobin concentration $(\mathrm{Hb})$, packed cell volume (PCV), and some red cell indices on Day 10. This was in line with an earlier report by Ejele et al. [9] and Umar et al. [10] though the drugs were administered singly and at variance with high PCV and leucocytes mean value reported by Odunukwe et al. [11].

There was no statistically significant difference $(P>0.05)$ in all the parameters evaluated when the test group were compared with the control on Day 25. The most probable explanation may be that in the test groups, the animals must have adapted or adjusted to its normal body physiology through a negative feedback mechanism, hence, must have compensated for the reduction in some blood parameters at the start of drug administration. However, this was disproved by a statistically significant decrease $(P<0.05)$ in the total WBC on Day 25 in groups $C$ and $E$ indicating that the drug may possess both dose- and time-dependent leucopenic inhibitory effects on the progenitor cells in the bone-marrow 
TABLE 5: Comparison of the mean \pm S.E of the haematological parameters after 25 days of HAART administration with the control group.

\begin{tabular}{|c|c|c|c|c|c|}
\hline Parameter & $\begin{array}{l}\text { Group A } \\
0 \mathrm{mg} / \mathrm{Kg}\end{array}$ & $\begin{array}{l}\text { Group B } \\
3 \mathrm{mg} / \mathrm{Kg}\end{array}$ & $\begin{array}{l}\text { Group C } \\
6 \mathrm{mg} / \mathrm{Kg}\end{array}$ & $\begin{array}{l}\text { Group D } \\
12 \mathrm{mg} / \mathrm{Kg}\end{array}$ & $\begin{array}{c}\text { Group E } \\
24 \mathrm{mg} / \mathrm{Kg}\end{array}$ \\
\hline $\mathrm{RBC}\left(\times 10^{12} / \mathrm{L}\right)$ & $7.52 \pm 0.69$ & $8.47 \pm 0.16$ & $8.95 \pm 0.20$ & $6.78 \pm 1.03$ & $6.42 \pm 0.75$ \\
\hline $\mathrm{HB}(\mathrm{g} / \mathrm{dL})$ & $14.55 \pm 1.05$ & $14.50 \pm 0.10$ & $16.20 \pm 0.50$ & $12.60 \pm 2.20$ & $11.55 \pm 1.35$ \\
\hline $\mathrm{PCV}(\mathrm{L} / \mathrm{L})$ & $53.20 \pm 3.10$ & $52.40 \pm 0.40$ & $59.60 \pm 2.70$ & $44.10 \pm 8.10$ & $40.05 \pm 4.75$ \\
\hline $\mathrm{MCHC}(\mathrm{g} / \mathrm{dL})$ & $27.50 \pm 0.50$ & $28.00 \pm 1.00$ & $27.50 \pm 0.50$ & $28.85 \pm 0.45$ & $28.90 \pm 0.10$ \\
\hline $\operatorname{MCV}(\mathrm{fL})$ & $71.20 \pm 2.20$ & $62.50 \pm 1.50$ & $66.70 \pm 4.50$ & $64.50 \pm 2.50$ & $62.65 \pm 0.35$ \\
\hline $\mathrm{MCH}(\mathrm{pg})$ & $19.40 \pm 0.40$ & $39.30 \pm 21.70$ & $18.15 \pm 0.95$ & $18.55 \pm 0.45$ & $18.00 \pm 1.00$ \\
\hline RETICS (\%) & $0.90 \pm 0.10$ & $1.20 \pm 0.10$ & $1.30 \pm 0.10$ & $1.10 \pm 0.10$ & $1.35 \pm 0.05$ \\
\hline PLAT $\left(\times 10^{9} / \mathrm{L}\right)$ & $624.50 \pm 113.50$ & $798.50 \pm 3.50$ & $791.00 \pm 29.00$ & $815.00 \pm 26.00$ & $798.50 \pm 13.05$ \\
\hline $\mathrm{WBC}\left(\times 10^{9} / \mathrm{L}\right)$ & $10.75 \pm 0.15$ & $8.25 \pm 0.65$ & $8.80 \pm 2.70$ & $8.40 \pm 0.30^{*}$ & $7.30 \pm 0.80^{*}$ \\
\hline NEUT (\%) & $28.00 \pm 8.00$ & $7.50 \pm 1.50$ & $13.50 \pm 5.50$ & $17.50 \pm 4.50$ & $7.50 \pm 0.50$ \\
\hline LYMPH (\%) & $72.00 \pm 8.00$ & $92.50 \pm 1.50$ & $86.50 \pm 5.50$ & $81.00 \pm 5.00$ & $92.50 \pm 0.50$ \\
\hline EOS $(\%)$ & $0.00 \pm 0.00$ & $0.00 \pm 0.00$ & $0.00 \pm 0.00$ & $0.00 \pm 0.00$ & $0.00 \pm 0.00$ \\
\hline MONO (\%) & $0.00 \pm 0.00$ & $0.00 \pm 0.00$ & $0.00 \pm 0.00$ & $1.50 \pm 0.50$ & $0.00 \pm 0.00$ \\
\hline BAS (\%) & $0.00 \pm 0.00$ & $0.00 \pm 0.00$ & $0.00 \pm 0.00$ & $0.00 \pm 0.00$ & $0.00 \pm 0.00$ \\
\hline
\end{tabular}

RBC: red blood cell, HB: haemoglobin concentration, PCV: packed cell volume, MCHC: mean cell haemoglobin concentration, MCV: mean cell volume, MCH: mean cell haemoglobin, RETICS: reticulocytes, PLAT: platelets, WBC: white blood cell, NEUT: neutrophils, LYMPH: lymphocytes, EOS: eosinophils, BAS: basophils.

*Compared with the control group $* P<0.05$.

TABLE 6: One way ANOVA of the mean \pm S.E haematological parameters of the rats administered $12 \mathrm{mg} / \mathrm{Kg}$ body weight (Group C) of HAART on Days 5, 10, 15, 20, and 25.

\begin{tabular}{|c|c|c|c|c|c|}
\hline Parameter & Day 5 & Day 10 & Day 15 & Day 20 & Day 25 \\
\hline $\mathrm{RBC}\left(\times 10^{12} / \mathrm{L}\right)$ & $7.23 \pm 0.13$ & $6.89 \pm 0.14$ & $9.14 \pm 0.61$ & $7.86 \pm 0.31$ & $8.95 \pm 0.20^{*}$ \\
\hline $\mathrm{HB}(\mathrm{g} / \mathrm{dL})$ & $12.55 \pm 0.25$ & $12.55 \pm 0.45$ & $14.55 \pm 1.15$ & $13.70 \pm 0.40$ & $16.20 \pm 0.50^{*}$ \\
\hline $\mathrm{PCV}(\mathrm{L} / \mathrm{L})$ & $39.00 \pm 1.00$ & $42.50 \pm 1.50$ & $57.40 \pm 5.40$ & $51.00 \pm 1.00$ & $59.60 \pm 2.70^{*}$ \\
\hline $\mathrm{MCHC}(\mathrm{g} / \mathrm{dL})$ & $32.00 \pm 1.20$ & $29.70 \pm 0.30$ & $25.50 \pm 0.50$ & $26.70 \pm 0.40$ & $27.50 \pm 0.50^{* *}$ \\
\hline $\mathrm{MCH}(\mathrm{pg})$ & $57.50 \pm 0.50$ & $62.00 \pm 1.00$ & $62.95 \pm 1.45$ & $65.30 \pm 1.70$ & $66.70 \pm 4.50$ \\
\hline MCV (fL) & $17.30 \pm 0.10$ & $18.15 \pm 0.25$ & $15.90 \pm 0.20$ & $17.45 \pm 0.15$ & $18.15 \pm 0.95$ \\
\hline RETICS (\%) & $1.30 \pm 0.30$ & $1.15 \pm 0.15$ & $1.60 \pm 0.20$ & $0.95 \pm 0.05$ & $1.30 \pm 0.10$ \\
\hline PLAT $\left(\times 10^{9} / \mathrm{L}\right)$ & $571.00 \pm 72.00$ & $370.00 \pm 232.00$ & $800.50 \pm 20.50$ & $787.50 \pm 1.50$ & $791.00 \pm 29.00^{* *}$ \\
\hline $\mathrm{WBC}\left(\times 10^{9} / \mathrm{L}\right)$ & $6.85 \pm 1.95$ & $9.30 \pm 1.10$ & $5.95 \pm 1.05$ & $7.65 \pm 2.05$ & $8.80 \pm 2.70$ \\
\hline NEUT (\%) & $20.00 \pm 4.00$ & $24.50 \pm 3.50$ & $27.00 \pm 3.00$ & $25.50 \pm 2.50$ & $13.50 \pm 5.50^{* * *}$ \\
\hline LYMPH (\%) & $78.50 \pm 3.50$ & $75.50 \pm 3.50$ & $72.00 \pm 4.00$ & $74.5 \pm 2.50$ & $86.50 \pm 5.50^{* * *}$ \\
\hline EOS (\%) & $0.50 \pm 0.50$ & $0.50 \pm 0.50$ & $0.00 \pm 0.00$ & $0.00 \pm 0.00$ & $0.00 \pm 0.00$ \\
\hline MONO (\%) & $1.00 \pm 1.00$ & $0.00 \pm 0.00$ & $1.00 \pm 1.00$ & $0.00 \pm 0.00$ & $0.00 \pm 0.00$ \\
\hline BAS (\%) & $0.00 \pm 0.00$ & $0.00 \pm 0.00$ & $0.00 \pm 0.00$ & $0.00 \pm 0.00$ & $0.00 \pm 0.00$ \\
\hline
\end{tabular}

RBC: red blood cell, HB: haemoglobin concentration, PCV: packed cell volume, MCHC: mean cell haemoglobin concentration, MCV: mean cell volume, MCH: mean cell haemoglobin, RETICS: reticulocytes, PLAT: platelets, WBC: white blood cell, NEUT: neutrophils, LYMPH: lymphocytes, EOS: eosinophils, BAS: basophils.

* Compared with the control group ${ }^{*} P<0.05,{ }^{* *} P<0.01, * * * P<0.001$.

as earlier reported by Umar et al. [10] or directly on the matured cells in the peripheral system.

There was a time-related statistically significant increase $(P<0.05)$ in the two major blood cells_- RBC and platelet count suggesting that administration of fixed-dose combination of HAART stimulated both erythropoietic and thrombopoietic activities by indirectly stimulating erythropoietin and thrombopoietin in the kidney or directly stimulating the pluripotential stem cells in the bone marrow. It could also be contested that the pancytopenia observed in HIV/AIDS patients may probably be due to either the virus infection or other latent malignancies not as a result of HAART administration.

Hence, from the result of this present study, it can be concluded that HAART when administered in fixed-dose combinations have no subacute haematotoxic effects. Its use in 
the treatment of HIV and other viral related infection should be encouraged and haematological parameters should be monitored.

\section{References}

[1] M. Dybul, A. S. Fauci, J. G. Bartlett, J. E. Kaplan, and A. K. $\mathrm{Pau}$, "Guidelines for using antiretroviral agents among HIVinfected adults and adolescents: the panel on clinical practices for treatment of HIV," Annals of Internal Medicine, vol. 137, no. 5, pp. 381-433, 2002.

[2] E. Daugas, J. P. Rougier, and G. Hill, "HAART-related nephropathies in HIV-infected patients," Kidney International, vol. 67, no. 2, pp. 393-403, 2005.

[3] P. G. Yeni, S. M. Hammer, C. C. J. Carpenter et al., "Antiretroviral treatment for adult HIV infection in 2002: updated recommendations of the international AIDS societyUSA panel," The Journal of the American Medical Association, vol. 288, no. 2, pp. 222-235, 2002.

[4] G. R. Serjeant, Y. Grandison, K. Mason, B. Sergeant, A. Sewell, and S. Vaidya, "Haematological indices in normal negro children: a Jamaican cohort from birth to five years," Clinical and Laboratory Haematology, vol. 2, no. 3, pp. 169-178, 1980.

[5] E. H. Karazawa and M. Jamra, "Normal hematologic parameters," Revista de Saude Publica, vol. 23, no. 1, pp. 58-66, 1989.

[6] D. M. Evans, I. H. Frazer, and N. G. Martin, "Genetic and environmental causes of variation in basal levels of blood cells," Twin Research, vol. 2, no. 4, pp. 250-257, 1999.

[7] G. M. Dal Colleto, D. W. Fulker, O. C. Barreto, and M. Koiya, "Genetic and environmental effects on blood cells," Acta Geneticae Medicae et Gemellologiae, vol. 42, no. 3-4, pp. 245-252, 1993.

[8] M. R. H. Taylor, C. V. Holland, R. Spencer, J. F. Jackson, G. I. O'Connor, and J. R. O’Donnell, "Haematological reference ranges for schoolchildren," Clinical and Laboratory Haematology, vol. 19, no. 1, pp. 1-15, 1997.

[9] O. A. Ejele, C. A. Erhabor, and A. Nwauche, "Haematologic effects of some antiretroviral drugs in patients with AIDS," Sahel Medical Journal, vol. 8, pp. 25-29, 2004.

[10] R. A. Umar, M. J. Ladan, S. W. Hassan, Y. Sa’id, A. Y. Abbas, and I. B. Oduolisaeme, "Administration of antiretroviral drugs (lamivudine, Nevirapine and Stavudine) has no untoward effect on haematological profile in Albino rats," Asian Journal of Biochemistry, vol. 2, no. 2, pp. 147-151, 2007.

[11] N. Odunukwe, N. E. O. Idigbe, T. A. Adewole, D. I. Onwuyekwe, and A. Audu, Abstract of Clinical Research, Federal Ministry of Health, Lagos, Nigeria. 

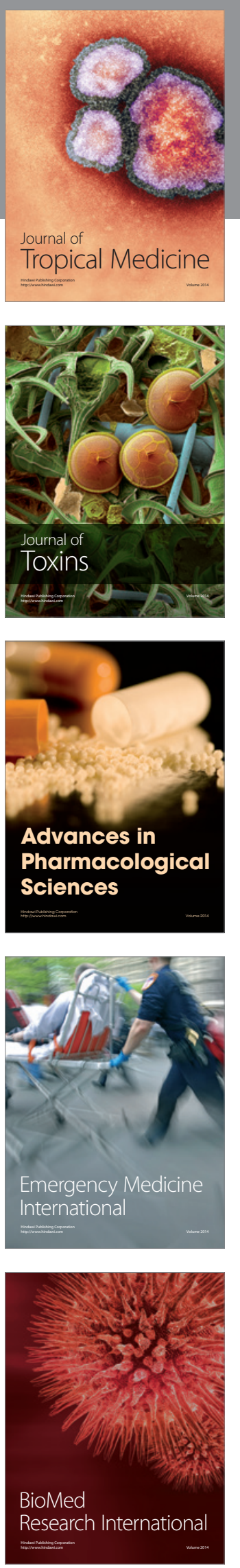
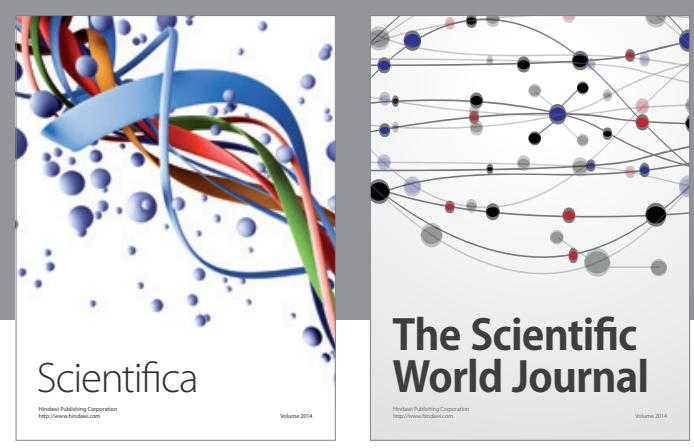

The Scientific World Journal
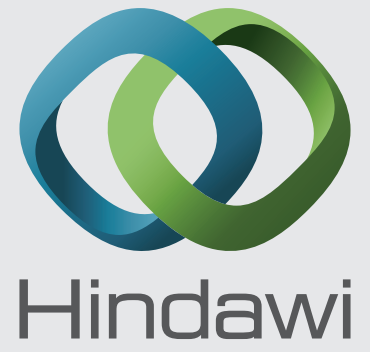

Submit your manuscripts at

http://www.hindawi.com
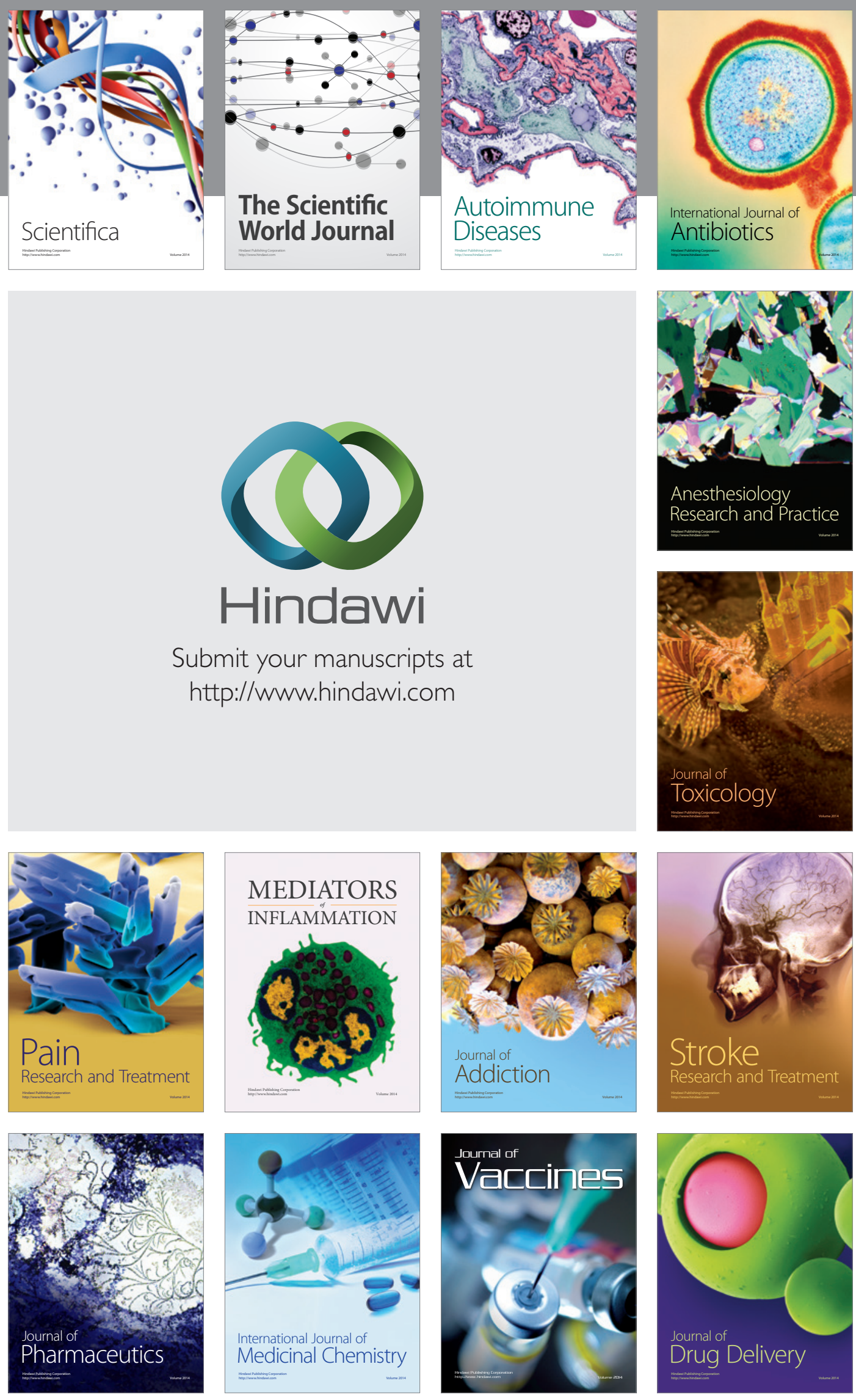